\title{
Measuring Shear Viscosity Using Correlations
}

\author{
Sean Gavin ${ }^{1}$ and Mohamed Abdel-Aziz ${ }^{2}$ \\ ${ }^{1}$ Department of Physics and Astronomy, Wayne State University, Detroit, MI, 48202, USA \\ ${ }^{2}$ Institut für Theoretische Physik, J.W. Goethe Universität, 60438 Frankfurt am Main, Germany
}

\section{Received on 15 December, 2006}

\begin{abstract}
Measurements of transverse momentum fluctuations can be used to determine the shear viscosity [1]. We use current data to estimate the viscosity-to-entropy ratio in the range from 0.08 to 0.3 , and discuss how future measurements can reduce this uncertainty.
\end{abstract}

Keywords: Relativistic Heavy Ions; Event-by-event fluctuations

\section{INTRODUCTION}

Elliptic flow measurements at RHIC are described by ideal viscosity-free hydrodynamics, indicating that the quark-gluon system produced in these collisions is a nearly perfect liquid [2-6]. In particular, the strong suppression of flow due to shear viscosity predicted by weak-coupling transport calculations is not observed [5]. This result is exciting because a small viscosity relative to the entropy density of the system may indicate that the system is more strongly coupled than expected: The collisional shear viscosity is proportional to the mean free path, which is shorter when the coupling is stronger.

But is the viscosity really small? Hirano et al. point out that color glass condensate formation may produce more elliptic flow than considered in refs. $[4,5]$, requiring a larger viscosity for agreement with data [8]. We therefore seek experimental information on viscosity that does not rely on elliptic flow.

We propose that transverse momentum correlation measurements can be used to extract information on the kinematic viscosity,

$$
\mathrm{v}=\eta / T s,
$$

where $\eta$ is the shear viscosity, $s$ is the entropy density and $T$ is the temperature. This ratio characterizes the strength of the viscous force relative to the fluid's inertia and, consequently, determines the effect of $\eta$ on the flow [6]. We argue that viscous diffusion broadens the rapidity dependence of transverse momentum correlations, and then show how these correlations can be extracted from measurements of event-by-event $p_{t}$ fluctuations.

A number of experiments have studied transverse momentum fluctuations at SPS and RHIC [9, 10]. Interestingly, the STAR collaboration reports a $60 \%$ increase of the relative rapidity width for $p_{t}$ fluctuations when centrality is increased [15]. While the STAR analysis differs from the one we propose, model assumptions provide a tantalizing hint that the viscosity is small.

Any experimental information on the kinematic viscosity of high energy density matter is vital for understanding the strongly interacting quark gluon plasma. Theorists had long anticipated a large collisional viscosity based on weak coupling QCD [16] and hadronic computations [17], with values of $\eta / s$ roughly of order unity for both phases near the crossover temperature $\sim 170 \mathrm{MeV}$. Supersymmetic Yang

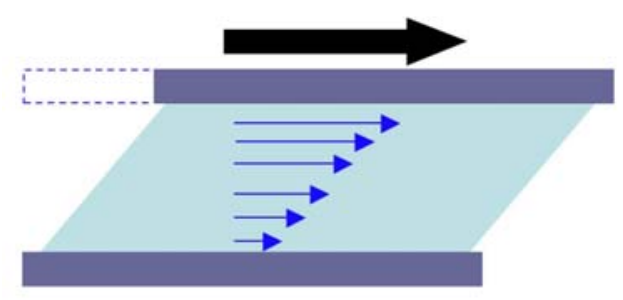

FIG. 1: (Color online) Shear flow results when plates move past one another.

Mills calculations give the significantly smaller ratio $\eta / s=$ $1 / 4 \pi$ in the strong coupling limit [18]. Lattice QCD calculations of the shear viscosity will eventually settle the question of the size of the viscosity near equilibrium [19]. However, the effective viscosity in the nonequilibrium ion-collision system may differ from these calculations. In particular, plasmainstability contributions can also explain the small viscosities in nuclear collisions [20].

We begin in the next section by formulating a simple model to illustrate how shear viscosity attenuates correlations due to fluctuations of the radial flow. In section III, we show how transverse momentum fluctuations can be used to measure these correlations. The magnitude of viscosity in heavy ion collisions at RHIC and SPS energies is discussed in section IV. In section V, we then demonstrate the impact of viscosity on the rapidity distribution of fluctuations. We explore the implications of current fluctuation data in section VI.

Before wading into the quark-gluon liquid, it is useful to recall how shear viscosity affects the flow of more common fluids. In a classic example of shear flow, a liquid is trapped between two parallel plates in the $x y$ plane, while one plate moves at constant speed in the $x$ direction as shown in fig. 1 . The fluid is pulled along with the plate, so that $v_{x}$ varies with the normal distance $z$. The viscous contribution to the stress energy tensor is then

$$
T_{z x}=-\eta \partial v_{x} / \partial z
$$

see ref. [21] for a general treatment.

To fix definitions, recall that relativistic hydrodynamic evolution satisfies the energy-momentum conservation law $\partial_{\mu} T^{\mu \nu}=0$. The stress-energy tensor is $T^{\mu \nu}=(e+P) U^{\mu} U^{\nu}-$ $P g^{\mu \nu}+\tau^{\mu \nu}$, where $e$ is the energy density, $P$ is the pressure, 


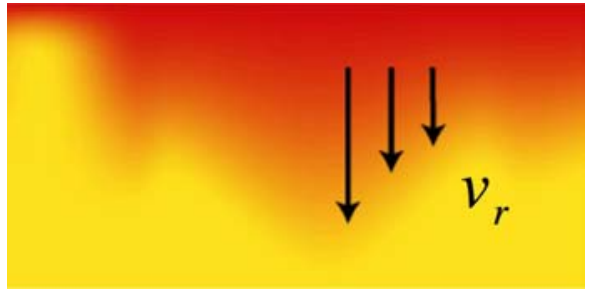

FIG. 2: (Color online) The transverse velocity deviates from the average by an amount that varies throughout the collision volume. Viscosity drives this deviation to zero.

$U^{\mu}=\gamma(1, \mathbf{v})$ is the four-velocity of the fluid, and $\gamma=(1-$ $\left.v^{2}\right)^{-1 / 2}$. The dissipative contribution is $\tau^{\mu v}$. Following Landau and Lifshitz, we define the four velocity to vanish when $T^{0 i}=0$ [21]. Then the momentum current is $T_{0 i}=\gamma^{2}(e+P) v_{i}$ and $\tau^{\mu v}$ depends only on the viscosity, with $\tau^{00}=0=\tau^{0 i}$ and

$$
\tau_{i j}=-\eta\left\{\nabla_{i} v_{j}+\nabla_{j} v_{i}-\frac{2}{3} \delta_{i j} \nabla \cdot \mathbf{v}\right\}-\zeta \delta_{i j} \nabla \cdot \mathbf{v}
$$

in a local rest frame. We mention that the bulk viscosity $\zeta$ resists compression or expansion in which $\nabla \cdot \mathbf{v} \neq 0$; the classic example is a Hubble-like expansion. Typically $\zeta \ll \eta[17]$; see however [23].

\section{VISCOSITY AND DIFFUSION}

Central nuclear collisions produce a high energy density fluid that flows outward with an average radial velocity $v_{r}$. In the hydrodynamic description of these collisions, we typically assume that $v_{r}$ varies smoothly with spacetime $(t, \mathbf{x})$ and is the same for all collisions of a fixed impact parameter. For central collisions, $v_{r}$ is cylindrically symmetric. More realistically, small deviations $\mathbf{u}(\mathbf{x})$ of the radial flow occur throughout the fluid, varying with each ion-collision event. Such deviations occur, e.g., because the number and location of nucleon-nucleon subcollisions varies in each event.

Viscous friction arises as neighboring fluid elements flow past each other as illustrated in fig. 2. This friction reduces $\mathbf{u}$, driving the velocity toward the local average $v_{r}$. The final size of the velocity increment $\mathbf{u}$ depends on the magnitude of the viscosity and the lifetime of the fluid.

In order to illustrate how the damping of radial flow fluctuations depends on the viscosity of the fluid, we introduce a velocity increment in the radial direction $u$ that depends only on the longitudinal coordinate $z$ and $t$. Our aim is to determine the linear response of the fluid to this perturbation. For simplicity, we take the unperturbed flow as slowly varying, and work in a co-moving frame where $v_{r}$ locally vanishes. As in (2), the flow of neighboring fluid elements at different radial speeds $u(z)$ produces a shear stress

$$
T_{z r}=-\eta \partial u / \partial z
$$

This stress changes the transverse momentum current of the fluid, which is generally

$$
T_{0 r}=\gamma^{2}(\varepsilon+p) v_{r}
$$

for energy density $\varepsilon$, pressure $p$, and $\gamma=\left(1-v^{2}\right)^{-1 / 2}$ [21]. The perturbation $u$ results in the change

$$
g_{t}(\mathbf{x})=\delta T_{0 r} \approx(\varepsilon+p) u
$$

in the co-moving frame, while

$$
\partial g_{t} / \partial t=-\partial T_{z r} / \partial z
$$

which follows from the energy-momentum conservation equation $\partial_{\mu} T^{\mu \nu}=0$.

We combine these results to obtain a diffusion equation for the momentum current

$$
\partial g_{t} / \partial t=v \nabla^{2} g_{t}
$$

to linear order. The kinematic viscosity is given by

$$
\nu=\eta /(\varepsilon+p) .
$$

This quantity measures the relative strength of the viscous relative to the fluid's and inertia, as is most apparent in the nonrelativistic limit, where $\varepsilon+p \rightarrow \rho$ for $\rho$ the mass density $(c=1)$. It follows that $\nu \approx \eta / \rho$ [21]. In our relativistic system at small net baryochemical potential, $\mu \approx 0$, so that $\varepsilon+p \approx T s$. Then $v$ is given by (1).

Observe that (8) applies for any fluctuation $\mathbf{g}_{t}$ for which $\nabla \cdot \mathbf{g}_{t}=0$ [21]; our physically-motivated radial $g_{t}(z, t)$ is a specific instance of such a flow. Such shear modes are related to sound waves (compression modes) but diffuse rather than propagate [22]. Note that the scale over which sound is attenuated $\Gamma_{s}=(4 \eta / 3+\zeta) / T s$ depends on both shear and bulk viscosity [21-23].

Viscosity tends to reduce fluctuations by distributing the excess momentum density $g_{t}$ over the collision volume. This effect broadens the rapidity profile of fluctuations. We write (8) in terms of the spatial rapidity $y=1 / 2 \ln (t+z) /(t-z)$ and proper time $\tau=\left(t^{2}-z^{2}\right)^{1 / 2}$ to find

$$
\partial g_{t} / \partial \tau=\left(v / \tau^{2}\right) \partial^{2} g_{t} / \partial y^{2}
$$

A similar equation is used to study net charge diffusion in ref. [26], and we can translate many of those results to the present context. Defining

$$
V \equiv\left\langle(y-\langle y\rangle)^{2}\right\rangle=\frac{\int y^{2} g_{t} d y}{\int g_{t} d y}
$$

for $\langle y\rangle=0$, we multiply both sides of (11) by $y^{2}$ and integrate to obtain

$$
\frac{d}{d \tau} V=\frac{2 v}{\tau^{2}}
$$

For a constant $v$, we compute the rapidity broadening

$$
\Delta V=\frac{2 v}{\tau_{o}}\left(1-\frac{\tau_{o}}{\tau}\right)
$$

where $\Delta V \equiv V-V\left(\tau_{o}\right)$ for $\tau_{o}$ the formation time. Observe that $V \rightarrow 2 v / \tau_{o}$ as $\tau \rightarrow \infty$. Also, note that the diffusion equation (10) can be solved directly to find $g_{t}(\tau, y) \propto$ 
$\exp \left[-y^{2} / 2 V(\tau)^{1 / 2}\right]$. The ratio (11) remains finite despite the fact that $g_{t} \rightarrow 0$ in that limit.

We briefly remark on the effect of the mean radial flow $v_{r}$ and transverse degrees of freedom on the growth of the rapidity width. In the comoving frame where $v_{r}$ is small, we can generalize (8) as

$$
\frac{\partial g_{t}}{\partial \tau}+\mathbf{v}_{r} \cdot \nabla_{\perp} g_{t}+\mathbf{g}_{t} \cdot \nabla_{\perp} v_{r}=\frac{v}{\tau^{2}}\left(\frac{\partial^{2}}{\partial y^{2}}+\nabla_{\perp}^{2}\right) g_{t} .
$$

We transform the diffusion equation (14) into (10) by replacing $g_{t}$ by a quantity that is averaged over the transverse coordinate $\mathbf{r}_{\perp}$. To see this, integrate both sides of (14) over $\mathbf{r}_{\perp}$. The transverse contributions lead to "surface terms" that vanish to this linear order. We stress that this result is independent of the azimuthal anisotropy of the collision volume. This result follows because $g_{t}$ is conserved - it doesn't matter where the momentum is in the transverse plane, as long as we add it up. Of course, that only applies if we limit our interest to the rapidity dependance. To study the azimuthal behavior of fluctuations we must solve the full eq. (14).

\section{CORRELATIONS AND DIFFUSION}

\section{A. Transverse momentum correlations}

We extend this discussion to address a more general ensemble of fluctuations by considering the correlation function

$$
r_{g}=\left\langle g_{t}\left(\mathbf{x}_{1}\right) g_{t}\left(\mathbf{x}_{2}\right)\right\rangle-\left\langle g_{t}\left(\mathbf{x}_{1}\right)\right\rangle\left\langle g_{t}\left(\mathbf{x}_{2}\right)\right\rangle .
$$

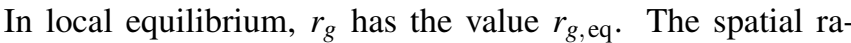
pidity dependence of $\Delta r_{g} \equiv r_{g}-r_{g, e q}$ is broadened by momentum diffusion. If the rapidity width of the one-body density follows (13), then the width of $\Delta r_{g}$ in the relative rapidity $y_{r}=y_{1}-y_{2}$ grows from an initial value $\sigma_{o}$ following

$$
\sigma^{2}=\sigma_{o}^{2}+2 \Delta V\left(\tau_{f}\right),
$$

where $\tau_{f}$ is the proper time at which freeze out occurs. This equation is entirely plausible, since diffusion spreads the rapidity of each particle in a given pair with a variance $\Delta V$. We then take

$$
\Delta r_{g}\left(y_{r}, y_{a}\right) \propto e^{-y_{r}^{2} / 2 \sigma^{2}-y_{a}^{2} / 2 \Sigma^{2}}
$$

where (16) gives the width in relative rapidity and the width in average rapidity $y_{a}=\left(y_{1}+y_{2}\right) / 2$ is $\Sigma$.

To compute fluctuation observables in the next sections, we identify spatial and momentum-space rapidity at a fixed freeze out proper time $\tau_{f}$. Observe that ISR and FNAL data on the rapidity dependence of multiplicity fluctuations [24] can be characterized as Gaussian near midrapidity. Moreover, these data show that charged particle correlations are functions of the relative rapidity $y_{r}$ with only a weak dependence on the average rapidity $y_{a}$. Correspondingly, we take $\Sigma \gg \sigma$. In the next section we see that diffusion increases both $\sigma$ and $\Sigma$ compared to their initial values $\sigma_{o}$ and $\Sigma_{o}$ at the hadronization time $\tau_{o}$ in accord with (25) and (26). For simplicity, we assume that $\Sigma_{o}$ is sufficiently large that we can neglect the time dependence of $\Sigma$ in (26).

\section{B. Transport in fluctuating systems}

Observe that (16) and (17) are exact for our diffusion model. To see that this is the case, we apply the theoretical framework developed by Van Kampen and others [25] to viscous diffusion. We have used this framework elsewhere to study the effect of diffusion on net charge fluctuations [26]. Since the key results of this section, (16) and (17), are "obvious," this section may be skipped on a first reading. However, the details are amusing.

The framework of ref. [25] generalizes the classic problem of Brownian motion, in which a dust particle wanders through a fluid with mean displacement $d$ that satisfies $d^{2} \sim$ $\left\langle\mathbf{x}^{2}\right\rangle-\langle\mathbf{x}\rangle^{2} \propto t$ on average. Microscopic molecular collisions cause the particle's motion, while friction of the macroscopic particle with the liquid dampens it. Here, we seek an evolution equation for $r_{g}$, in which $g_{t}(\mathbf{x}, t)$ replaces the Brownian $\mathbf{x}$. Our equation for $r_{g}$ must have the correct limit $r_{g, e q}$ when the system is in equilibrium. Equation (33) in the next section implies that

$$
\begin{aligned}
r_{g, e q}\left(\mathbf{x}_{1}, \mathbf{x}_{2}\right) & =\delta\left(\mathbf{x}_{1}-\mathbf{x}_{2}\right) \int f\left(\mathbf{x}_{1}, \mathbf{p}\right) p_{t}^{2} d p \\
& =n\left\langle p_{t}^{2}\right\rangle \delta\left(\mathbf{x}_{1}-\mathbf{x}_{2}\right),
\end{aligned}
$$

where $f(\mathbf{x}, \mathbf{p})$ is the phase space distribution of particles, $n$ is the density.

In the previous section we found that $g_{t}$ obeys a diffusion equation (8) in the regime of small fluctuations, but this statement requires clarification. Consider an ensemble of systems with different initial $g_{t}$. At the macroscopic scales of interest, viscosity indeed dampens fluctuations, working to drive $g_{t}(\mathbf{x}, t) \rightarrow 0$. Opposing this dampening are Brownian-like velocity and thermal fluctuations due to the microscopic motion and collisions of particles. These fluctuations are present at all times prior to freeze out. In equilibrium, fluctuations and viscous dissipation are balanced.

To describe the effect of these fluctuations on the momentum current, we treat $g_{t}$ as a stochastic quantity. It is the average of $g_{t}$ over our ensemble that satisfies (8); such an ensemble average is always implicit in hydrodynamics. Going further, we write a Langevin equation for the fluctuating $g_{t}$ as

$$
\frac{\partial g_{t}}{\partial t}+\nabla \cdot \mathbf{j}_{g}=0
$$

where $\mathbf{j}_{g}=-v \nabla g_{t}+\mathbf{k}$ and $\mathbf{k}$ is a stochastic contribution to the current. If we divide the fluid into tiny cells with a macroscopic number of particles, then particle and momentum flow into and out of each cell gives rise to k. Observe that (19) conserves the transverse momentum current in each 'event' in the ensemble. The current $\mathbf{k}$ is a Langevin noise term satisfying $\langle\mathbf{k}\rangle=0$ and

$$
\left\langle k_{i}\left(\mathbf{x}_{1}, t\right) k_{j}\left(\mathbf{x}_{2}, t^{\prime}\right)\right\rangle=K\left(\mathbf{x}_{1}, t\right) \delta_{i j} \delta\left(\mathbf{x}_{1}-\mathbf{x}_{2}\right) \delta\left(t_{1}-t_{2}\right) ;
$$

see [25]. The coefficient $K$ is determined by the requirement that fluctuations have the correct value in equilibrium (the fluctuation-dissipation theorem). 
Applying the methods in [25] to (19), we find that the correlation function (15) satisfies the diffusion equation

$$
\left(\frac{\partial}{\partial t}-v\left(\nabla_{1}^{2}+\nabla_{2}^{2}\right)\right) \Delta r_{g}\left(\mathbf{x}_{1}, \mathbf{x}_{2}\right)=0
$$

where $\Delta r_{g}=r_{g}-r_{g, e q}$. This equation means that over time $r_{g} \rightarrow r_{g, e q}$ as small deviations in the momentum current diffuse over the collision volume. As for (8), we emphasize that (21) only applies in the linear regime where fluctuations are small.

To get a rough understanding of how (21) comes about, consider the change $\delta g_{t}$ in the time increment $\delta t$. The change of the two-point equal-time function at points $1 \equiv\left(\mathbf{x}_{1}, t\right)$ and $2 \equiv\left(\mathbf{x}_{2}, t\right)$ is $\delta\left\langle g_{t}(1) g_{t}(2)\right\rangle=\left\langle\delta g_{t}(1) g_{t}(2)\right\rangle+\left\langle g_{t}(1) \delta g_{t}(2)\right\rangle+$ $\left\langle\delta g_{t}(1) \delta g_{t}(2)\right\rangle$, where the increment $\delta g_{t}=\left(\partial g_{t} / \partial t\right) \delta t$ is given by (19). In the absence of noise, the term $\left\langle\delta g_{t}(1) \delta g_{t}(2)\right\rangle$ would vanish as $\delta t^{2}$. However, noise forces $g_{t}$ to undergo a "random-walk" so that $\delta g_{t}$ receives a stochastic increment $\propto \delta t^{1 / 2}$. This increment contributes to $\left\langle\delta g_{t}(1) \delta g_{t}(2)\right\rangle \propto \delta t$. One finds

$$
\left(\frac{\partial}{\partial t}-v\left(\nabla_{1}^{2}+\nabla_{2}^{2}\right)\right) r_{g}(1,2)=\nabla_{1} \cdot \nabla_{2}\langle K(1) \delta(1-2)\rangle ;
$$

the right side is zero in the absence of noise. Choosing $K$ so that $r_{g} \rightarrow r_{g, e q}$ for a uniform system in equilibrium gives (21).

The correlation function $\Delta r_{g}\left(y_{1}, y_{2}, \tau\right)$ then obeys

$$
\left(\frac{\partial}{\partial \tau}-\frac{\nu}{\tau^{2}} \frac{\partial^{2}}{\partial y_{1}^{2}}-\frac{\nu}{\tau^{2}} \frac{\partial^{2}}{\partial y_{2}^{2}}\right) \Delta r_{g}=0 .
$$

We write (23) in terms of the relative rapidity $y_{r} \equiv y_{1}-y_{2}$ and average rapidity $y_{a}=\left(y_{1}+y_{2}\right) / 2$ :

$$
\left(\frac{\partial}{\partial \tau}-\frac{2 v}{\tau^{2}} \frac{\partial^{2}}{\partial y_{r}^{2}}-\frac{v}{\tau^{2}} \frac{\partial^{2}}{\partial y_{a}^{2}}\right) \Delta r_{g}=0 ;
$$

the " 2 " follows from the transformation to relative rapidity $y_{r}$. To compute the widths of $\Delta r_{g}\left(y_{r}, y_{a}, \tau\right)$ in relative or average rapidity, one multiplies (24) by $y_{r}^{2}$ or $y_{a}^{2}$ and integrates over both variables. We find

$$
\Delta\left\langle\left(y_{r}-\left\langle y_{r}\right\rangle\right)^{2}\right\rangle=2 \Delta V(\tau)
$$

and

$$
\Delta\left\langle\left(y_{a}-\left\langle y_{a}\right\rangle\right)^{2}\right\rangle=\Delta V(\tau),
$$

where $\Delta V(\tau)$ is calculated in the previous section.

\section{VISCOSITY IN ION COLLISIONS}

Gyulassy and Hirano survey computations of the ratio of the shear viscosity to the entropy and find that both the hadron gas and the perturbative quark gluon plasma have $\eta / s \sim 1$, if one naively extrapolates these calculations near $T_{C}$ [6]. These values correspond to $v=\eta / T s$ roughly of order $1 \mathrm{fm}$ for $T_{C}=170 \mathrm{MeV}$. On the other hand, they argue that the entropy

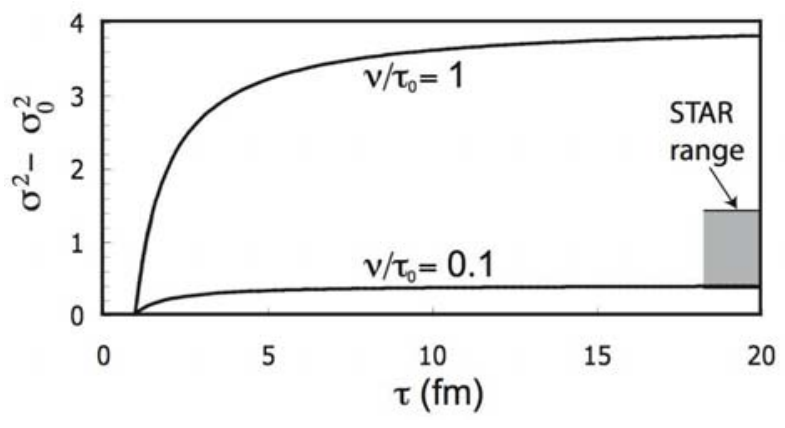

FIG. 3: Rapidity spread vs. time for momentum diffusion from (13) and (16) for two viscosity values. The gray area marks the range extrapolated from data in ref. [15] using (42).

increase near $T_{C}$ reduces $\eta / s$ for a strongly interacting plasma, perhaps to the supersymmetric Yang-Mills value $\eta / s=1 / 4 \pi$.

Motivated by these estimates, we show in fig. 3 the increase of $\sigma$ given by (13) and (16) as a function of $\tau$. Calculations for two values $v / \tau_{o} \sim 0.1$ and 1 schematically exhibit the likely range of viscous broadening. For $\tau_{o} \sim 1 \mathrm{fm}$, these values respectively correspond to $\eta / s \sim 1 / 4 \pi$ and 1 . We provide these calculations as benchmarks; more realistically, $v$ would effectively increase with $\tau$ depending on the state of the fluid.

We stress that the rapidity width depends on the viscous diffusion coefficient integrated over the collision lifetime. Comparing the viscous and perfect scenarios in fig. 3, we see that the largest contribution to this width comes from the earliest times. Consequently, we expect measurements of this width to yield information on the viscosity when the evolution is dominated by partons.

\section{FLUCTUATIONS AND CORRELATIONS}

\section{A. Transverse momentum covariance}

Variation of the radial fluid velocity over the collision volume induces correlations in the transverse momenta $p_{t}$ of particles [27]. To describe such correlations, we observe that an inhomogeneous fluid near local thermal equilibrium can be divided into cells that are small enough to be regarded as uniform, while containing a macroscopic number of particles. Particles emerging from cells of different radial velocity $v_{r}$ are more likely to have different $p_{t}$ than particles from the same cell. We describe the number of particles of momentum $\mathbf{p}$ in a cell at position $\mathbf{x}$ at the instant of freeze out as $d n=f(\mathbf{x}, \mathbf{p}) d p d x$, where $d p \equiv d^{3} p /(2 \pi)^{3}$ and $d x \equiv d^{3} x$. We take the phase space density $f(\mathbf{x}, \mathbf{p})$ to be a Boltzmann distribution corresponding to the fluid velocity $\mathbf{v}(\mathbf{x})$ and temperature $T(\mathbf{x})$. The temperature and velocity profiles both vary from event to event. A similar formulation is used in [28] to compute nonequilibrium $p_{t}$ fluctuations. Here, we focus on central collisions, where there is a body of RHIC data suggesting that local equilibrium is likely achieved.

To characterize the dynamic correlations of $p_{t}$, we use the 
transverse momentum covariance

$$
\mathcal{C}=\langle N\rangle^{-2}\left\langle\sum_{i \neq j} p_{t i} p_{t j}\right\rangle-\left\langle p_{t}\right\rangle^{2}
$$

where $i$ labels particles from each event and the brackets represent the event average. The average transverse momentum is

$$
\left\langle p_{t}\right\rangle \equiv\left\langle\sum p_{t i}\right\rangle /\langle N\rangle
$$

This quantity vanishes in local thermal equilibrium, where the momenta are uncorrelated and particle number fluctuations satisfy Poisson statistics, so that $\left\langle N^{2}\right\rangle-\langle N\rangle^{2}=\langle N\rangle$. It follows that $\left\langle p_{t 1} p_{t 2}\right\rangle_{\mathrm{eq}}=\langle N(N-1)\rangle\left\langle p_{t}\right\rangle^{2} /\langle N\rangle^{2}=\left\langle p_{t}\right\rangle^{2}$.

This covariance is related to the spatial correlations of the momentum current (15) by

$$
\mathcal{C}=\langle N\rangle^{-2} \int \Delta r_{g}\left(\mathbf{x}_{\mathbf{1}}, \mathbf{x}_{2}\right) d x_{1} d x_{2}
$$

To obtain this result, observe that near local equilibrium $f(\mathbf{x}, \mathbf{p})=\langle f\rangle+\delta f$, where the average distribution is $\langle f(\mathbf{x}, \mathbf{p})\rangle$ and the event-wise deviation $\delta f$ is necessarily small. Then

$$
\begin{aligned}
\langle N\rangle\left\langle p_{t}\right\rangle & =\left\langle\int p_{t} d n\right\rangle \\
& =\int p_{t}\langle f\rangle d p d x+\int\left\langle g_{t}(\mathbf{x})\right\rangle d x \\
& \equiv \int p_{t}\langle f\rangle d p d x .
\end{aligned}
$$

The contribution of fluctuations to the momentum current,

$$
g_{t}(\mathbf{x})=\int \delta f(\mathbf{x}, \mathbf{p}) p_{t} d p,
$$

vanishes on event averaging. Similarly, the unrestricted sum is

$$
\begin{aligned}
\left\langle\sum p_{t i} p_{t j}\right\rangle & =\left\langle\int p_{t 1} p_{t 2} d n_{1} d n_{2}\right\rangle \\
& =\langle N\rangle^{2}\left\langle p_{t}\right\rangle^{2}+\int\left\langle g_{t}\left(\mathbf{x}_{1}\right) g_{t}\left(\mathbf{x}_{2}\right)\right\rangle d x_{1} d x_{2} .
\end{aligned}
$$

We find

$$
\begin{aligned}
\int r_{g} d x_{1} d x_{2} & =\left\langle\sum_{\text {all } i, j} p_{t i} p_{t j}\right\rangle-\langle N\rangle^{2}\left\langle p_{t}\right\rangle^{2} \\
& =\langle N\rangle^{2} C+\left\langle\sum p_{t i}^{2}\right\rangle ;
\end{aligned}
$$

the second equality follows from (27). In local equilibrium, $\mathcal{C} \equiv 0$ implies $\int r_{g, \text { eq }} d x_{1} d x_{2}=\left\langle\sum p_{t i}^{2}\right\rangle$. Subtracting this term from (33) gives (29).

\section{B. Multiplicity variance}

The correlation information probed by $\mathcal{C}$ differs from that found in the multiplicity variance

$$
R=\left(\left\langle N^{2}\right\rangle-\langle N\rangle^{2}-\langle N\rangle\right) /\langle N\rangle^{2} .
$$

Multiplicity fluctuations and their relation to correlation functions are discussed in [29]. As before, and in accord with [29], we write

$$
R=\langle N\rangle^{-2} \int \Delta r_{n} d x_{1} d x_{2},
$$

where $\Delta r_{n}=r_{n}-r_{n, \text { eq }}$ and

$$
r_{n}=\left\langle n\left(\mathbf{x}_{1}\right) n\left(\mathbf{x}_{2}\right)\right\rangle-\left\langle n\left(\mathbf{x}_{1}\right)\right\rangle\left\langle n\left(\mathbf{x}_{2}\right)\right\rangle
$$

The density correlation function (36) carries different information than (15) because particle number is not conserved. Density fluctuations evolve by the full hydrodynamic equations, while $g_{t}$ follows diffusion. We mention that

$$
r_{n, e q}=\left\langle n\left(\mathbf{x}_{1}\right)\right\rangle \delta\left(\mathbf{x}_{1}-\mathbf{x}_{2}\right)
$$

which is analogous to (18) but somewhat easier to understand. Equation (37) implies that (36) vanishes when particle number fluctuations obey Poisson statistics, a property that is evident from (34).

\section{Rapidity dependance of $p_{t}$ covariance}

Viscosity information can be obtained from $\mathcal{C}$ as follows. For simplicity, we identify spatial and momentum space rapidity. The broadening in rapidity of $\Delta r_{g}$ depends on the shear viscosity via (16). Equation (29) implies that the rapidity dependence of $\Delta r_{g}$ can be measured by studying the dependence of (27) on the rapidity window in which particles are measured.

We illustrate this acceptance dependence in fig. 2 for the $\mathrm{v} / \tau_{o}$ values from fig. 1 as follows. The covariance $C$ is computed by integrating $\Delta r_{g}$ over an interval $-\Delta / 2 \leq y_{1}, y_{2} \leq \Delta / 2$ corresponding to the experimental acceptance. We use (17), (29), and assume $\Sigma \gg \Delta / 2$ to obtain

$$
\begin{aligned}
\langle N\rangle \mathcal{C} & \approx \frac{1}{\langle N\rangle} \int_{-\Delta / 2}^{\Delta / 2} \int_{-\Delta / 2}^{\Delta / 2} \Delta r_{g}\left(y_{1}, y_{2}\right) d y_{1} d y_{2} \\
& \approx \frac{2}{\langle N\rangle} \int_{0}^{\Delta / 2} d y_{a} \int_{-\Delta / 2+y_{a}}^{\Delta / 2-y_{a}} \Delta r_{g}\left(y_{r}, y_{a}\right) d y_{r} \\
& \approx\langle N\rangle C_{\infty} \operatorname{erf}(\Delta / \sqrt{8} \sigma),
\end{aligned}
$$

where the total number of charged particles is $\langle N\rangle \propto \Delta$ and $\langle N\rangle \mathcal{C}_{\infty}$ is the value obtained for a large rapidity window. We assume $\tau_{f} / \tau_{o} \sim 20$.

\section{CURRENT DATA}

\section{A. Rapidity dependence of $p_{t}$ fluctuations}

Transverse momentum fluctuations have been measured at STAR and at the CERN SPS. We now ask whether information from these measurements can provide any information on the viscosity. The covariance $\mathcal{C}$ we propose is sensitive 


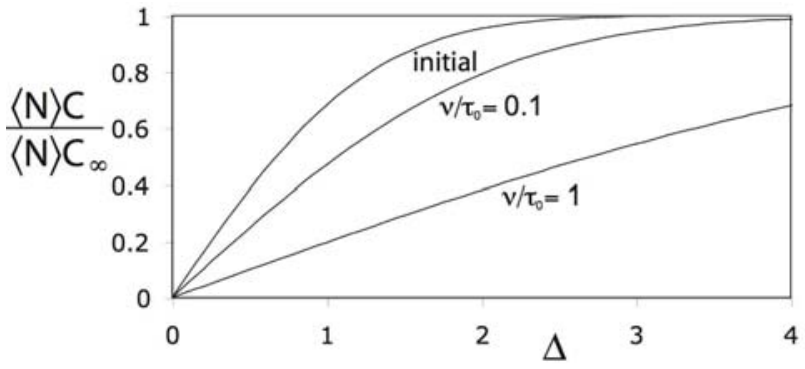

FIG. 4: Rapidity dependence of the $p_{t}$ covariance (27) for $\tau_{f} / \tau_{o} \sim$ 20. The initial distribution has $\sigma_{o} \sim 0.5$.

to the variation of the $p_{t}$ of particles as well as their number density, in that both quantities affect the momentum current. Other measures of $p_{t}$ fluctuations in the literature are designed to minimize any density contribution, often termed "volume fluctuations" [31]. This difference is important for the following discussion.

The STAR analysis in ref. [15] incorporates some of these ideas and, intriguingly, finds a broadening in rapidity together with a narrowing in azimuth for $p_{t}$ correlations in central compared to peripheral collisions. We will use the rapidity information to estimate the viscosity. However, the measured quantities differ sufficiently from $\mathcal{C}$ that this estimate requires significant model assumptions. We therefore regard the result only as a signal of our method's promise.

STAR employs the transverse momentum fluctuation observable $\Delta \sigma_{p_{t}: n}^{2}$ to construct a correlation function as a function of rapidity and azimuthal angle. They find that near-side correlations in azimuth are broadened in relative rapidity, with a rapidity width $\sigma_{*}$ that increases from roughly 0.45 in the most peripheral collisions to 0.75 in central ones [15]. In our terms,

$$
\Delta \sigma_{p_{t}: n}^{2}=\langle N\rangle^{-1}\left\langle\sum_{i \neq j}\left(p_{t i}-\left\langle p_{t}\right\rangle\right)\left(p_{t j}-\left\langle p_{t}\right\rangle\right)\right\rangle .
$$

To relate this to our observables, we expand the right side and use (28) to find

$$
\langle N\rangle \Delta \sigma_{p_{t}: n}^{2}=\left\langle\sum_{i \neq j} p_{t i} p_{t j}\right\rangle-\langle N(N-1)\rangle\left\langle p_{t}\right\rangle^{2},
$$

since the average number of pairs is $\langle N(N-1)\rangle$. We then obtain

$$
\frac{\Delta \sigma_{p_{t}: n}^{2}}{\langle N\rangle}=C-\left\langle p_{t}\right\rangle^{2} R,
$$

where $C$ and $R$ are respectively given by (27) and (34).

The quantity $\Delta \sigma_{p_{t}: n}^{2}$ therefore depends on both momentum current and density correlation functions (15) and (36),

$$
\Delta \sigma_{p_{t}: n}^{2}=\langle N\rangle^{-1} \int\left\{\Delta r_{g}-\left\langle p_{t}\right\rangle^{2} \Delta r_{n}\right\} d y_{1} d y_{2}
$$

We can directly compare $\sigma_{*}$ to $\sigma$ in fig. 1 if $\Delta r_{g}$ and $\Delta r_{n}$ have the same widths. Equation (16) then implies that the widths in central and peripheral collisions satisfy

$$
\sigma_{c}^{2}-\sigma_{p}^{2}=4 v\left(\tau_{f, p}^{-1}-\tau_{f, c}^{-1}\right) .
$$

Observe that the dependence on $\tau_{o}$ cancels. Taking the freeze out times in central and peripheral collisions to be $\tau_{f, c} \sim 20 \mathrm{fm}$ and $\tau_{f, p} \sim 1 \mathrm{fm}$, we then find $v \sim 0.09 \mathrm{fm}$. The value $\tau_{f, p} \sim 1 \mathrm{fm}$ is reasonable, since ref. [15] argues that the average participant path length is about $1 \mathrm{fm}$ for these peripheral collisions. We use (1) to find $\eta / s \sim 0.08$.

This result is remarkably close to the supersymmetric Yang Mills value $1 / 4 \pi$, and is consistent with some hydrodynamic comparisons to elliptic flow data [5]. However, we must be cautious: If $\Delta r_{g}$ and $\Delta r_{n}$ have different rapidity widths $\sigma$ and $\sigma_{n}$ then their relation to $\sigma_{*}$ depends on the relative strength of these contributions. Data in ref. [32] may indicate that $\sigma_{n}$ is roughly twice $\sigma_{*}$. As we argue shortly, $\sigma$ is bounded by $\sigma_{n}$ and $\sigma_{*}$. At the maximum value $\sigma=2 \sigma_{*}$, our dynamic assumptions yield $\eta / s=0.3$. Together, our estimates constitute an uncertainty range for the viscosity-to-entropy ratio, $0.08<\eta / s<0.3$. We also indicate the range of $\sigma_{c}^{2}-\sigma_{p}^{2}$ implied by the STAR data in fig. 3 as a gray band corresponding to $\sigma_{*}<\sigma<2 \sigma_{*}$.

To show $\sigma$ is bounded by $\sigma_{n}$ and $\sigma_{*}$, we observe that (42) implies that $\sigma_{*}$ follows from a distribution in relative rapidity

$$
f\left(y_{r}\right)=\Delta r_{g}-\left\langle p_{t}\right\rangle^{2} \Delta r_{n} .
$$

It follows that

$$
\sigma_{*}^{2}=\frac{\int y_{r}^{2} f\left(y_{r}\right)}{\int f\left(y_{r}\right)}=\frac{\sigma^{2} G-\sigma_{n}^{2}\left\langle p_{t}\right\rangle^{2} N}{G-\left\langle p_{t}\right\rangle^{2} N},
$$

where $G=\int \Delta r_{g}$ and $N=\int \Delta r_{n}$. We then find

$$
\sigma^{2}=\frac{\sigma_{*}^{2}+\beta \sigma_{n}^{2}}{1+\beta}
$$

where $\beta=\left\langle p_{t}\right\rangle^{2} N / G$. Although $\beta$ is not measured, the width cannot exceed $\sigma_{n} \sim 2 \sigma_{*}$. (We restrict our integrals to a rapidity and $p_{t}$ region where a Gaussian parameterization of $\Delta r_{g}$ and $\Delta r_{n}$ makes sense; hence $\beta \geq 0$ ).

We briefly comment on the narrowing in azimuth for $p_{t}$ correlations observed in ref. [15] in central compared to peripheral collisions. The diffusion broadening of azimuthal correlations is compensated by a narrowing due to the mean radial flow $v_{r}$, which hurls particles outward, and surface bias, which hides particles that flow in the wrong direction. Openingangle effects in resonance decays lead to further narrowing. These effects are familiar in HBT. To address the azimuthal dependence of correlations, an equation analogous to (24) can be derived from (14). One must also account for the azimuthal anisotropy of the collision volume.

\section{B. Other experimental information}

As an aside, we now note some of the information from other experimental $p_{t}$ fluctuation studies. The observable $\left\langle\delta p_{t 1} \delta p_{t 2}\right\rangle$ studied in [28] and measured in [9], [10] and [11] satisfies

$$
\Delta \sigma_{p_{t}: n}^{2}=\frac{\langle N(N-1)\rangle}{\langle N\rangle}\left\langle\delta p_{t 1} \delta p_{t 2}\right\rangle,
$$




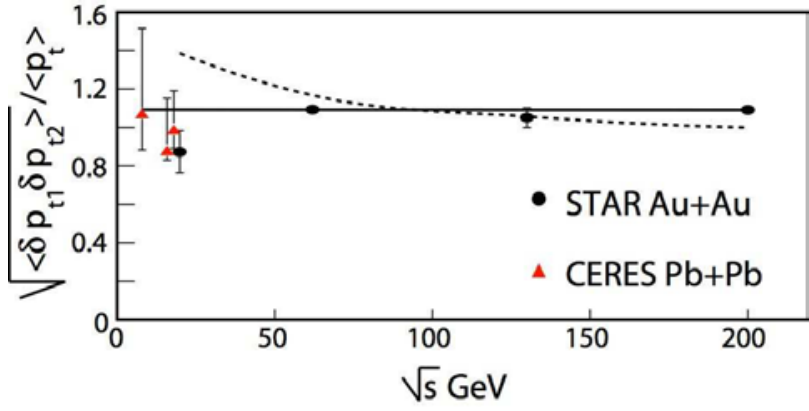

FIG. 5: (Color online) Energy dependence of the $p_{t}$ fluctuations using the observable (47). Data are from [9], [10], and [11].

where $R=\left(\left\langle N^{2}\right\rangle-\langle N\rangle^{2}-\langle N\rangle\right) /\langle N\rangle^{2}$ measures multiplicity fluctuations [29]. The energy dependence of this quantity is shown in fig. 5.

The energy independence of these measurements supports the hypothesis that the largest contribution to $p_{t}$ fluctuations is from the collective behavior of soft and semi-hard particles rather than jets as sometimes noted [34]. New PHENIX energy-dependence data further supports the soft origin of fluctuations [35]. Observe that (47) implies that $\Delta \sigma_{p_{t}: n}^{2}$ will increase with energy in proportion to the multiplicity if $\left\langle\delta p_{t 1} \delta p_{t 2}\right\rangle$ is energy dependent. All this concerns the overall scale of the fluctuations. Little can be said about the energy dependence of the rapidity distribution of these fluctuations at present.

Alternative observables $\Phi_{p_{t}}, F_{p_{t}}$, and $\Delta \sigma_{p_{t}}$ proposed in [30], [13], and [14] satisfy $F_{p_{t}} \approx \Phi_{p_{t}} / \sigma \approx\langle N\rangle\left\langle\delta p_{t 1} \delta p_{t 2}\right\rangle / 2 \sigma^{2}$ for $\sigma^{2}=\left\langle p_{t}^{2}\right\rangle-\left\langle p_{t}\right\rangle^{2}$, see e.g. [31]. Broadly, the differences in these various quantities is important only in peripheral collisions where the multiplicity is small. The different quantities have depend differently on the experimental efficiency, e.g., $\left\langle\delta p_{t 1} \delta p_{t 2}\right\rangle$ is designed to minimize efficiency dependence. Other differences may reflect the different experimental acceptance and analysis issues.

\section{SUMMARY}

In summary, we find that shear viscosity can broaden the rapidity correlations of the momentum current. This broadening can be observed by measuring the transverse momentum covariance (27) as a function of rapidity acceptance. Our rough estimate from current data, $\eta / s \sim 0.08-0.3$, is small compared to perturbative computations [6]. To reduce the experimental uncertainty, we suggest measuring $C$ to allow more direct access to the momentum density correlation function.

We stress that there is additional theoretical uncertainty in this estimate, mainly due to our freeze out model. In practice, $\sigma_{c}^{2}-\sigma_{p}^{2} \approx 4 v \tau_{f, p}^{-1}$, since $\tau_{f, p} \ll \tau_{f, c}$. The freeze out time in peripheral collisions $\tau_{f, p}$ is not plausibly smaller than our value $1 \mathrm{fm}$ (the nucleon radius), but may be twice as large. This would double the upper limit of our uncertainty band. That added uncertainty can eventually be reduced by measuring $\tau_{f, p}$ as in [33]. HBT and resonance effects omitted here may contribute only at the $10 \%$ and $15 \%$ levels, respectively [10]. Minijets, color glass, and other particle production effects modify $\sigma_{o}$ in (16). We assume that any modification cancels in studying the centrality dependence at fixed beam energy. As to our hydrodynamic treatment, we have not considered alternative transport formulations that enforce causality [36]. Furthermore, our linearized diffusion model of flow fluctuations is physically reasonable but highly idealized. A more refined hydrodynamic description will be necessary to confront the measurements we suggest.

How can we reduce the theoretical uncertainty? The viscosity of a common fluid can be measured by applying a known pressure and observing the resulting flow in a fixed geometry, e.g., a pipe. Alternatively, one can study the attenuation of high frequency sound waves from a calibrated source. Efforts to compare flow measurements to viscous hydrodynamic calculations are analogous to the first method [5]. Our observable $\mathcal{C}$ is in the spirit of ultrasonic attenuation. The early dynamics produces a spectrum of fluctuations analogous to sound waves that are attenuated by viscosity [37]. We suggest that experimenters pursue both approaches to extract quantitative viscosity information from ion collisions, since the initial conditions and model parameters are all unknown.

\section{Acknowledgements}

We thank J. Dunlop for kindly bringing the data in ref. [15] to our attention, and R. Bellwied, M. Gyulassy, G. Moschelli, and C. Pruneau for useful discussions. This work was supported in part by a U.S. National Science Foundation PECASE/CAREER award under grant PHY-0348559 (S.G.) and BMBF, GSI and DAAD (M.A-A.).
[1] S. Gavin and M. Abdel-Aziz, Phys. Rev. Lett. 97, 162302 (2006).

[2] I. Arsene et al. [BRAHMS Collaboration], Nucl. Phys. A 757, 1 (2005); K. Adcox et al. [PHENIX Collaboration], ibid. 184; B. B. Back et al. [PHOBOS Collaboration], ibid. 28; J. Adams et al. [STAR Collaboration], ibid. 102.

[3] K. H. Ackermann et al. [STAR Collaboration], Phys. Rev. Lett. 86, 402 (2001); C. Adler et al. [STAR Collaboration], Phys. Rev. Lett. 87, 182301 (2001); Phys. Rev. Lett. 89, 132301
(2002); Phys. Rev. C 66, 034904 (2002); J. Adams et al. [STAR Collaboration], Phys. Rev. C 72, 014904 (2005); K. Adcox et al. [PHENIX Collaboration], Phys. Rev. Lett. 89, 212301 (2002); S. S. Adler et al. [PHENIX Collaboration], Phys. Rev. Lett. 91, 182301 (2003); B. B. Back et al. [PHOBOS Collaboration], Phys. Rev. Lett. 89, 222301 (2002); Phys. Rev. Lett. 94, 122303 (2005); Phys. Rev. C 72, 051901 (2005).

[4] P. F. Kolb, P. Huovinen, U. W. Heinz, and H. Heiselberg, Phys. Lett. B 500, 232 (2001); P. Huovinen and P. V. Ruuskanen, 
arXiv:nucl-th/0605008.

[5] D. Teaney, Phys. Rev. C 68, 034913 (2003); J. Phys. G 30, S1247 (2004).

[6] T. Hirano and M. Gyulassy, Nucl. Phys. A 769, 71 (2006).

[7] L. P. Csernai, J. I. Kapusta, and L. D. McLerran, Phys. Rev. Lett. 97, 152303 (2006).

[8] T. Hirano, U. W. Heinz, D. Kharzeev, R. Lacey, and Y. Nara, Phys. Lett. B 636, 299 (2006).

[9] C. Pruneau, Proc. Quark Matter 2005, in press; J. T. Mitchell, J. Phys. G 30, S819 (2004).

[10] J. Adams et al. [STAR Collaboration], Phys. Rev. C 72, 044902 (2005).

[11] D. Adamova et al. [CERES Collaboration], Nucl. Phys. A 727, 97 (2003).

[12] T. Anticic et al. [NA49 Collaboration], Phys. Rev. C 70, 034902 (2004).

[13] S. S. Adler et al. [PHENIX Collaboration], Phys. Rev. Lett. 93 092301 (2004).

[14] J. Adams et al. [STAR Collaboration], Phys. Rev. C 71, 064906 (2005).

[15] J. Adams et al. [STAR Collaboration], J. Phys. G 32, L37, (2006); arXiv:nucl-ex/0509030.

[16] A. Hosoya and K. Kajantie, Nucl. Phys. B 250, 666 (1985); P. Danielewicz and M. Gyulassy, Phys. Rev. D 31, 53 (1985); G. Baym, H. Monien, C. J. Pethick, and D. G. Ravenhall, Phys. Rev. Lett. 64, 1867 (1990); H. Heiselberg, Phys. Rev. D 49 , 4739 (1994); P. Arnold, G. D. Moore, and L. G. Yaffe, JHEP 0011, 001 (2000); 0305, 051 (2003).

[17] S. Gavin, Nucl. Phys. A 435, 826 (1985); M. Prakash, M. Prakash, R. Venugopalan, and G. Welke, Phys. Rept. 227, 321 (1993); A. Muronga, Phys. Rev. C 69, 044901 (2004); S. Muroya and N. Sasaki, Prog. Theor. Phys. 113, 457 (2005).

[18] P. Kovtun, D. T. Son, and A. O. Starinets, Phys. Rev. Lett. 94, 111601 (2005); 87, 081601 (2001).

[19] A. Nakamura and S. Sakai, arXiv:hep-lat/0510039.

[20] M. Asakawa, S. A. Bass, and B. Muller, Phys. Rev. Lett. 96, 252301 (2006).

[21] L. D. Landau and E. M. Lifshitz, Fluid Mechanics (Pergamon,
Oxford, 1959).

[22] D. M. Forster, Hydrodynamic fluctuations, Broken symmetry, and Correlation Functions, (Benjamin, New York, 1975).

[23] K. Paech and S. Pratt, arXiv:nucl-th/0604055.

[24] J. Whitmore, Phys. Rept. 27, 187 (1976); L. Foa, Physics Reports, 22, 1 (1975); H. Boggild and T. Ferbel, Ann. Rev. Nucl. Part. Sci. 24, 451 (1974).

[25] N. G. Van Kampen, Stochastic Processes in Physics and Chemistry, (Elsevier Science, Amsterdam, 1997); C. W. Gardiner, Handbook of Stochastic Methods for Physics, Chemistry and the Natural Sciences, (Springer, Berlin, 2002).

[26] M. Abdel-Aziz and S. Gavin, Phys. Rev. C 70, 034905 (2004).

[27] S. A. Voloshin, Phys. Lett. B 632, 490 (2006).

[28] S. Gavin, Phys. Rev. Lett. 92, 162301 (2004); J. Phys. G 30, S1385 (2004).

[29] C. Pruneau, S. Gavin and S. Voloshin, Phys. Rev. C 66, 044904 (2002).

[30] M. Gazdzicki and S. Mrowczynski, Z. Phys. C 54, 127 (1992).

[31] S. A. Voloshin, V. Koch, and H. G. Ritter, Phys. Rev. C 60, 024901 (1999).

[32] J. Adams et al. [STAR Collaboration], Phys. Rev. C 73, 064907 (2006).

[33] M. Lisa, Acta Phys. Polon. B 35, 37 (2004); C. Markert, J. Phys. G 31, S169 (2005).

[34] M. Abdel-Aziz and S. Gavin, Nucl. Phys. A 774, 623 (2006).

[35] J. T. Mitchell, Proc. Quark Matter 2006.

[36] A. Muronga, Phys. Rev. Lett. 88, 062302 (2002); Phys. Rev. C 69, 034903 (2004); A. Muronga and D. H. Rischke, arXiv:nuclth/0407114; U. W. Heinz, H. Song, and A. K. Chaudhuri, Phys. Rev. C 73, 034904 (2006); A. K. Chaudhuri, Phys. Rev. C 74, 044904 (2006); R. Baier, P. Romatschke, and U. A. Wiedemann, Phys. Rev. C 73, 064903 (2006); R. Baier and P. Romatschke, arXiv:nucl-th/0610108.

[37] The fluctuations probed by our $p_{t}$ observable are shear modes, which are not strictly sound waves; see the discussion following eq. (1). 